\title{
ARTICLE
}

\section{Major bacterial lineages are essentially devoid of CRISPR-Cas viral defence systems}

David Burstein', Christine L. Sun ${ }^{1,2}$, Christopher T. Brown ${ }^{3}$, Itai Sharon', Karthik Anantharaman', Alexander J. Probst ${ }^{1}$, Brian C. Thomas ${ }^{1} \&$ Jillian F. Banfield ${ }^{1,4}$

Current understanding of microorganism-virus interactions, which shape the evolution and functioning of Earth's ecosystems, is based primarily on cultivated organisms. Here we investigate thousands of viral and microbial genomes recovered using a cultivationindependent approach to study the frequency, variety and taxonomic distribution of viral defence mechanisms. CRISPR-Cas systems that confer microorganisms with immunity to viruses are present in only $10 \%$ of 1,724 sampled microorganisms, compared with previous reports of $40 \%$ occurrence in bacteria and $81 \%$ in archaea. We attribute this large difference to the lack of CRISPR-Cas systems across major bacterial lineages that have no cultivated representatives. We correlate absence of CRISPR-Cas with lack of nucleotide biosynthesis capacity and a symbiotic lifestyle. Restriction systems are well represented in these lineages and might provide both non-specific viral defence and access to nucleotides.

\footnotetext{
${ }^{1}$ Department of Earth and Planetary Science, University of California, Berkeley, Berkeley, California 94720, USA. ${ }^{2}$ Department of Microbiology and Immunology, Stanford University School of Medicine, Stanford, California 94305, USA. ${ }^{3}$ Department of Plant and Microbial Biology, University of California, Berkeley, Berkeley, California 94720, USA. ${ }^{4}$ Department of Environmental Science, Policy and Management, University of California, Berkeley, California 94720, USA. Correspondence and requests for materials should be addressed to J.F.B. (email: jbanfield@berkeley.edu).
} 
$\mathrm{M}$ icroorganisms play fundamental roles in the functioning of the biosphere, yet their existence depends on their ability to resist viral predation. CRISPR (clustered regularly interspaced short palindromic repeats) - Cas ( mechanisms that confer bacteria and archaea with acquired immunity to viruses. The two main parts of the system are a CRISPR array, composed of spacers matching foreign DNA flanked by repeats, and an operon of cas genes that encode for proteins that process the CRISPR array and cleave DNA targeted by the spacers ${ }^{1-3}$. Due to its high specificity and programmable nature, CRISPR-Cas has been harnessed to develop a powerful new genome editing technology ${ }^{4-6}$. Current knowledge of the frequency and distribution of CRISPR-Cas is based primarily on genomes of isolated microorganisms ${ }^{3,7}$. Here, we expand the investigation of the prevalence, variety and taxonomic distribution of these systems to include more than 40 major lineages of uncultivated bacteria and archaea. We also investigate CRISPR-Cas locus transfer over large evolutionary distances and the presence of alternative viral resistance mechanisms. Finally, we consider possible implications for a link between symbiotic lifestyles and absence of this system.

\section{Results}

Sampling of microbial and viral populations in groundwater. We studied groundwater filtrates collected as part of a field experiment designed to reproduce conditions that enriched for microbes from lineages that are referred to as Candidate Phyla (CP) because they lack isolated representatives ${ }^{8}$. Over 14 weeks, acetate was added to groundwater in an aquifer adjacent to the Colorado River, near the town of Rifle, Colorado, USA ${ }^{9}$. Twelve samples were collected before, during and after acetate addition. DNA was extracted from cells recovered on serial $0.2 \mu \mathrm{m}$ (post $1.2 \mu \mathrm{m}$ ) and $0.1 \mu \mathrm{m}$ (post $0.2 \mu \mathrm{m}$ ) filters and sequenced using Illumina HiSeq technology. In total, we generated more than $250 \mathrm{Gbp}$ of DNA sequence information. The $0.1 \mu \mathrm{m}$ filtrate samples were of particular interest, as these fractions were enriched in CP bacteria, uncultivated archaea and viruses ${ }^{9-11}$. The acetate manipulation shifted community composition over time, providing access to a wide variety of microorganisms and viruses. We reconstructed 867 circularized viral genomes (614 non-redundant sequences) and more than $57 \mathrm{Mbp}$ of linear or partial virus sequences. Further, we sampled 1,724 microbial genomes that, according to analyses of rRNA genes and phylogenetically informative proteins, originated from members of over 40 phyla, including at least $20 \mathrm{CP}^{9,11}$ (Supplementary Data 1). Essentially all of the sampled organisms are from previously unstudied lineages, novel at the genus or higher taxonomic levels.

CRISPR-Cas systems are less prevalent than expected. CRISPR arrays were identified within the reconstructed genomes (Supplementary Data 2), and their spacers were used to search the metagenomic data set for their viral targets. Given the coexistence of viruses and their potential microbial hosts in the sampled groundwater ecosystem, we anticipated that a large fraction of the CRISPR spacers would match viral sequences. Within the microbial draft genomes we identified CRISPR arrays with 156 distinct repeats harbouring 3,874 unique spacer sequences. Despite the large sampling of viral sequences, only 172 distinct CRISPR spacers $(<5 \%)$ could be assigned to targets with high probability ( $\geq 90 \%$ identity). Further, only 14 circularized viral genomes $(<3 \%)$ were targeted by a spacer. These findings suggested that CRISPR-Cas systems might be less important in environmental microbial communities than expected based on current understanding of the prevalence of this defence system.

We next identified cas operons using hidden Markov models built based on previously described profiles for the encoded Cas proteins ${ }^{7}$. The cas operons were present in merely 166 out of 1,724 draft genomes: in $9.7 \%$ of the sampled bacteria and in $9.3 \%$ of the archaea (Fig. 1a and Supplementary Data 3). In comparison, previous analyses based primarily on sequences from cultivated microorganisms indicate CRISPR-Cas occurrence in $40 \%$ of bacteria and in $81 \%$ of archaea ${ }^{3,7}$. The difference between the previously reported incidence rate and that found here could arise to a certain extent from the partial nature of the draft genomes recovered from the metagenomic samples. To account for this, we normalized for genome incompleteness, as reflected by the inventory of expected single-copy genes ${ }^{9,12}$. After this correction, the frequency at which CRISPR-Cas systems were detected in microorganisms from the studied subsurface system was still substantially below current expectation: $10.4 \%$ in bacteria and $10.1 \%$ in archaea. We calculated the probability that the difference between the observed occurrence rate of bacterial cas operons and the expected occurrence rate based on previous reports is due to genome incompleteness. The probability that cas 1 was present in $40 \%$ of bacterial genomes but missed in $29.6 \%$ of the cases due to genome incompleteness was estimated to be $<10^{-90}$ (using a conservative estimation; see Methods). These results are compelling because they are based on more than 1,700 draft genomes, an unprecedentedly large environmental data set.

Entire bacterial phyla essentially lack CRISPR-Cas. Strikingly, CRISPR-Cas systems were absent in almost all complete and draft genomes of organisms from four bacterial phyla and two superphyla, most of which are part of the Candidate Phyla Radiation $(\mathrm{CPR})^{11}$. Specifically, they were found in only $1.4 \%$ of over 354 high quality draft genomes ( $\geq 80 \%$ complete) from organisms assigned to the superphyla Parcubacteria (OD1) and Microgenomates (OP11), and the phyla WWE3, Berkelbacteria (ACD58), WS6 and TM6 (which is not part of the CPR). We do not attribute the absence of these proteins to sequence divergence since our model-based approach was able to identify cas operons in those lineages in a few cases. Further, we considered CRISPRCas to be present even if only a CRISPR array was found. To rule out the possibility that incorrect gene predictions obscured identification of cas operons, we confirmed this analysis using six-frame translations of all genome sequences. This did not reveal any additional operons. The low incidence of CRISPR-Cas systems was not typical for other bacterial groups sampled in this study (Fig. 1a). In fact, we found CRISPR-Cas systems in $31.9 \%$ of the sampled bacterial genomes belonging to the phyla that were represented in the last major CRISPR-Cas survey ${ }^{7}$. This is close to the $40 \%$ occurrence rate reported in that survey, indicating that the low abundance of CRISPR-Cas we observe is primarily due to the consideration of novel genomes from multiple phyla that were not included in previous surveys.

Samples from additional environments were examined to determine whether the same lineages lack CRISPR-Cas systems in those environments as well. We investigated genomes ( $\geq 70 \%$ complete) reconstructed from metagenomic samples taken at Crystal Geyser, a deep subsurface $\mathrm{CO}_{2}$-saturated coldwater geyser located on the east bank of Green River, UT, USA. Out of 42 genomes of Parcubacteria, Microgenomates, WWE3 and Berkelbacteria species recovered from this site, only three (7.1\%) had CRISPR-Cas (Supplementary Data 4). We further analysed all 32 publically available draft and high quality genomes from organisms belonging to these lineages. These organisms were sampled from a variety of environments, including a 
a

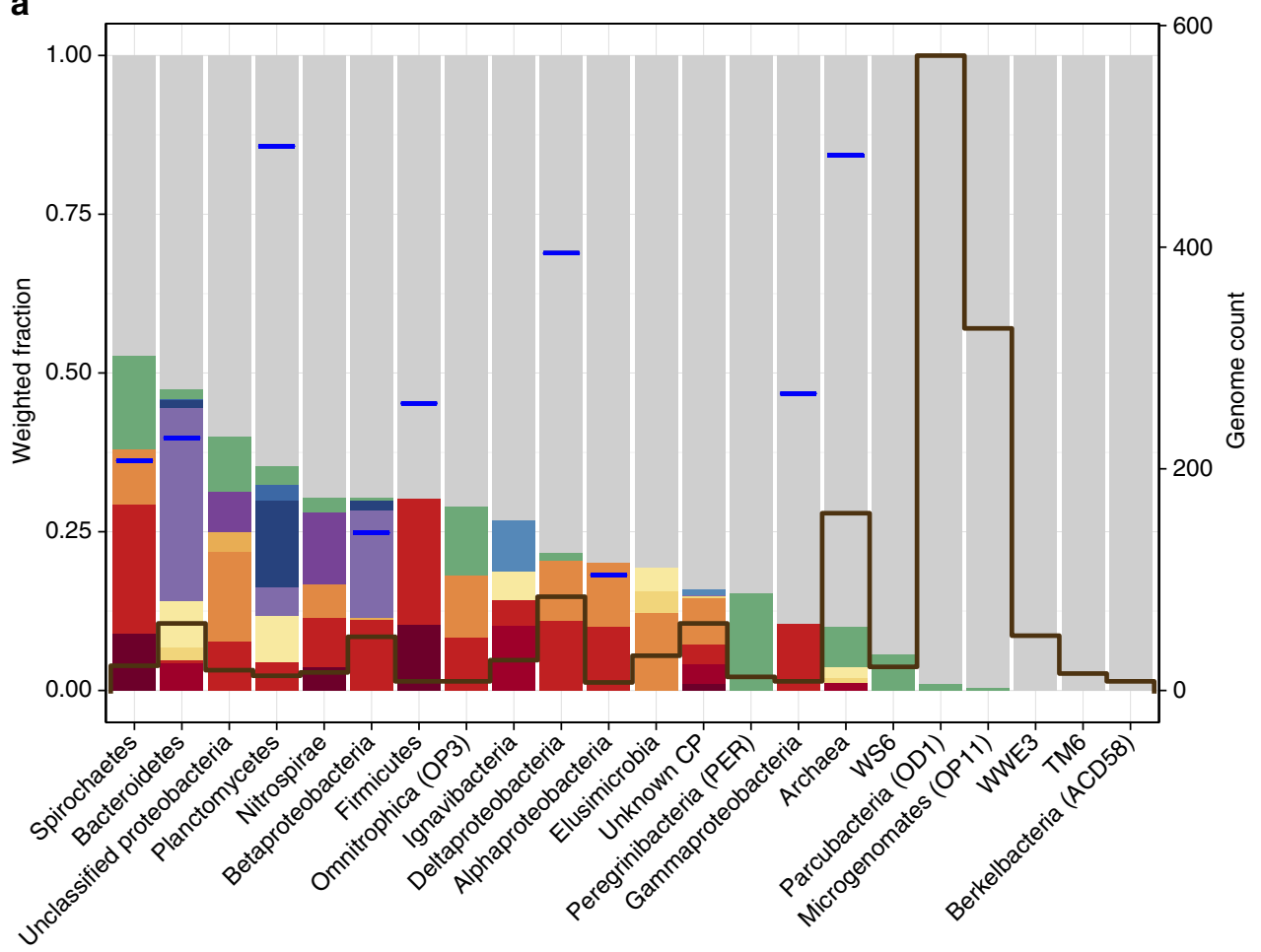

b

\begin{tabular}{|c|c|c|c|c|c|}
\hline \multicolumn{6}{|c|}{ Cas subtype } \\
\hline I-A & I-E & II-A & III-A & U-? & \multirow{2}{*}{$\begin{array}{l}\text { Compared } \\
\text { NCBI Cas } \\
\text { frequency }\end{array}$} \\
\hline I-B & I-F & II-B & III-B & $?-?$ & \\
\hline I-C & I-U & II-? & III-U & None & Genome \\
\hline I-D & I-? & & III-? & & count \\
\hline
\end{tabular}

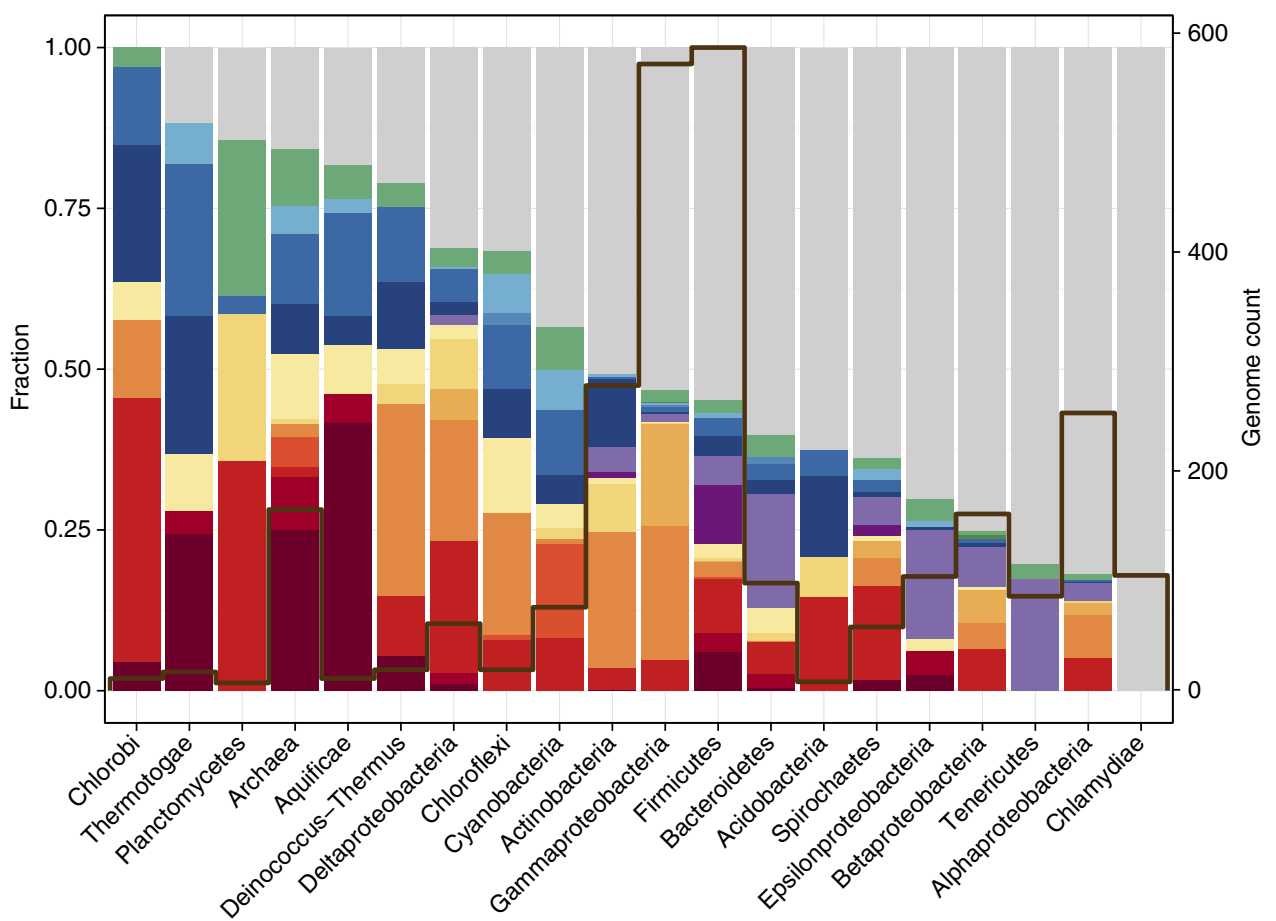

Figure 1 | The frequency of cas operons in different lineages. (a) Rifle groundwater metagenomic data sets and (b) NCBI complete bacterial and archaeal genomes. Only lineages with more than ten genomes are presented. Column heights are normalized for genome completeness, column colours represent CRISPR-Cas type, and the bold brown line represents the number of genomes. Blue vertical lines in a represent the fraction of genomes with CRISPR-Cas among NCBI complete genomes for applicable lineages. Two superphyla and four phyla in the groundwater samples, including the two most abundant ones, superphyla Parcubacteria (OD1), and Microgenomates (OP11), have an extremely low incidence of cas operons. 
hospital sink biofilm, a deep-sea hydrothermal vent, a geothermal spring, a deep underground mine and more (Supplementary Data 4). None of these genomes encoded for a CRISPR-Cas systems. Collectively, these results support our finding that major CP lineages are essentially devoid of CRISPR-Cas defence systems.

To place these results in context, we used the same methods to perform an updated survey that included all 2,740 publically available complete bacterial and archaeal genomes from NCBI (Supplementary Data 5) to determine the incidence of CRISPR-Cas systems. The analysis indicated an incidence rate of $40.6 \%$ for bacterial genomes and $79.4 \%$ for archaeal genomes (Supplementary Data 6), in agreement with previous reports ${ }^{3,7}$. Interestingly, we did not detect any cas operons or CRISPR arrays in the Chlamydiae phylum. It has been previously reported that Chlamydiae have no type II CRISPR-Cas systems ${ }^{13}$, but here we report that all 105 complete genomes from organisms representing this phylum lack any type of CRISPR-Cas system. This finding reinforces the conclusion that these defence systems can be essentially absent from entire bacterial lineages. Overall, our analyses suggest that the distribution of the CRISPR-Cas immune system is highly uneven across the different branches of the bacterial tree.

Evidence for horizontal transfer of CRISPR-Cas across phyla. The near-absence of CRISPR-Case across entire phyla is intriguing given the evidence that these systems are readily transferred between microbes, potentially even across phylum boundaries $^{14-18}$. In support of cross-phyla transfer, we find that the phylogeny of Cas1 proteins is inconsistent at the phylum level with the taxonomy of the organisms that contain the system (Fig. 2). The results for Cas1 and repeat sequences are congruent, as expected if simultaneous transfer of both locus components is required to maintain CRISPR-Cas function. Analyses of both Cas1 and the repeats suggest that CRISPR-Cas was transferred to CPR organisms, but in most cases selected against, as indicated by partial cas operons (Supplementary Data 3) and the restricted distribution of the system among these organisms. Notably, we identified $100 \%$ identical repeat sequences in organisms from three different bacterial phyla (Fig. 2). Overall, these findings demonstrate that horizontal transfer of the CRISPR-Cas indeed occurs frequently and even among organisms from different phyla, but in certain phyla it is selected against.

CRISPR-Cas systems are classified into different types based on the inventory of Cas proteins ${ }^{7}$. As in genomes from cultivated organisms, the least common system type in Rifle groundwater samples was type II. This type is characterized by minimal cas operons and the presence of Cas9, a protein of great biotechnological interest. Cas9 was found in organisms from only four bacterial phyla, and in $13.6 \%$ of all cas operons. Due to the interest in Cas9 we provide sequences and phylogeny of Cas9 proteins identified in Rifle metagenomic samples (Supplementary Data 7; Supplementary Fig. 1).

Lack of CRISPR-Cas in symbionts. We considered the possibility that CRISPR-Cas systems are absent from specific phyla because these organisms experience little viral predation. However, we identified the presence of integrated viral genes, as well as alternative viral defence systems, mostly restrictionmodification $^{19}$ and abortive infection ${ }^{20}$, in the CP lineages lacking CRISPR-Cas (Supplementary Fig. 2A). Further indicating ongoing viral predation, a recent cryo-TEM characterization of $0.1 \mu \mathrm{m}$ filtrates from the current study detected virus particles directly associated with the surfaces of the ultra-small cells, primarily from Parcubacteria (OD1), Microgenomates (OP11) and WWE3 (ref. 10). In addition, several Chlamydia viruses have been reported ${ }^{21}$. Given evidence that these organisms experience viral predation, we conclude that CRISPR-Cas is probably selected against because the costs of maintaining the systems ${ }^{22,23}$ outweighs the benefit of the defence it provides.

One of the potentially detrimental costs associated with CRISPR-Cas viral defence is autoimmunity, which arises when CRISPR arrays acquire spacers that target the host genome ${ }^{24}$. Interestingly, two of the nine arrays found in CP with low CRISPR-Cas incidence had spacers with significant identity to the host bacterial genome. In one case, we found a spacer $100 \%$ identical to a segment of the $16 \mathrm{~S}$ ribosomal RNA gene. This observation indicates that avoidance of self-targeting might be ineffective in these organisms, thus rendering CRISPR-Cas toxic to these cells.

Alternatively, it is possible that CRISPR-Cas systems are not retained due to shared features of the organisms that lack them. Notably, organisms from CPR, TM6 and Chlamydiae have very small genomes $(\sim 1 \mathrm{Mbp})$ and they are inferred to be obligate symbionts ${ }^{11,25-29}$. Thus, we hypothesized that selection against CRISPR-based viral defence is a general feature of symbionts. To test this, we compared the presence of CRISPR-Cas among wellstudied free-living and obligate symbionts (genus-level analysis, see Methods and Supplementary Data 8). We found a significant association $\left(P\right.$ value $<1.6 \times 10^{-8}$ ANOVA) between absence of CRISPR-Cas and obligate symbiotic lifestyles (Fig. 3). To support previous reports of symbiotic lifestyle of $\mathrm{CPR}^{11,25-28}$ organisms, we surveyed 528 high-quality genomes from the groundwater samples (estimated completeness $\geq 80 \%$ ) for presence of fattyacid and nucleotide biosynthesis. All the organisms from phyla lacking CRISPR-Cas had no fatty-acid initiation or elongation pathways (Supplementary Fig. 3), suggesting they acquire fatty acids from external sources. An analysis of the co-occurrence of nucleotide biosynthesis pathways with viral defence systems in a combined set of quality genomes from NCBI and the groundwater samples, revealed that CRISPR-Cas systems are extremely underrepresented in organisms without nucleotide biosynthesis pathways ( $P$ value $<10^{-116}$, Fisher exact test, Supplementary Fig. 4A). Curiously, among organisms lacking nucleotide biosynthesis pathways, restriction systems were more represented in CPR organisms (78\%) compared with non-CPR organisms $(62 \%)$. Similarly, the number of restriction enzymes encoded in the genomes of CPR organisms lacking nucleotide biosynthesis is significantly higher than in non-CPR organisms lacking these pathways ( $P$ value $<10^{-39}$, Mann-Whitney test, Supplementary Fig. 4B).

\section{Discussion}

Entire lineages of uncultivated organisms, which have been shown to be widespread across many environments ${ }^{10,30}$, are essentially devoid of CRISPR-Cas. We suggest three possible explanations for this observation. The first explanation arises from their likely obligate symbiotic lifestyle, an inference based on the lack of biosynthesis pathways for basic cellular building blocks, and supported by a recent experimental study showing that a CPR organism from the Saccharibacteria (TM7) phylum is an obligate epibiont of an Actinobacterium ${ }^{31}$. A lifestyle that requires close association of multiple symbiont cells with a host cell could lead to higher phage densities, which have been experimentally shown by Westra et al. ${ }^{22}$ to cause selection against CRISPR-Cas. Second, the efficacy of CRISPR-Cas depends on the viral mutation rate and spacer incorporation frequency ${ }^{32}$. Given their low ribosome contents ${ }^{10}$, CPR probably have extremely slow growth rates, and may not be able to mount a response to an infection event fast enough or to acquire spacers quickly enough for the CRISPR-Cas system to be effective. 


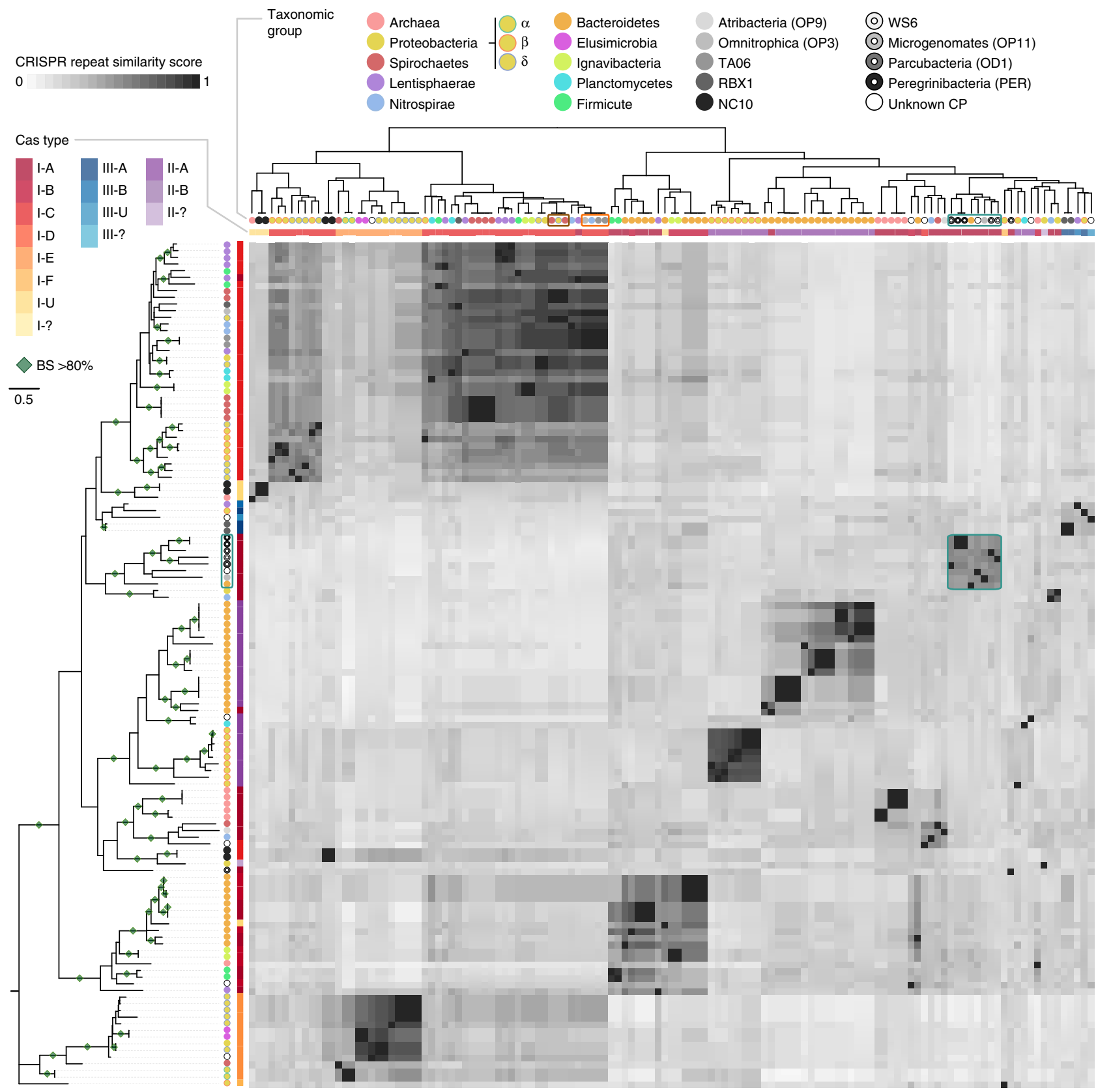

Figure 2 | Cas1 phylogeny versus repeat similarity in CRISPR-Cas retrieved from Rifle groundwater samples. Cas1 phylogeny (left) compared with the similarity of repeats in associated CRISPR arrays (top). Coloured circles on the branch tips represent taxonomic affiliation. Colour strip represent CRISPR-Cas type. Heat map shades correspond to full-length repeat similarity. Genomes from different phyla with identical repeats are marked by brown and orange boxes. The cyan box highlights a cluster of genomes with closely related Cas1 proteins and similar repeats, which includes one Bacteroidetes genome and most of the genomes from CPR organisms that have a CRISPR-Cas system. Green diamonds mark branching with bootstrap values (BS) $>80 \%$.

Finally, the CRISPR-Cas system may be selected against due to autoimmunity ${ }^{24,33}$ : self-targeting spacers have been incorporated, possibly due to lack of mechanisms to distinguish self from other ${ }^{34}$ in these small genomes.

Genes for restriction enzymes, which are overrepresented in these genomes compared with symbionts from other lineages, may provide access to nucleotides by degrading exogenous DNA, including that derived from inactivated viruses. Lipids may be acquired from the hosts of these organisms, as was previously suggested for the intracellular pathogen Renibacterium salmonarum $^{35}$. Combined, these findings raise the intriguing possibility of symbioses due to mutual benefits that arise from membrane sharing and DNA acquisition from degraded viruses. Specifically, CPR that are extracellular symbionts of bacteria, and derive cell wall material from their host bacteria may retain the surface-associated viral recognition targets of the host cell. It is possible then that CPR bacteria provide a service to their hosts by serving as 'viral decoys', lowering the host viral load, while taking advantage of the degraded viral DNA as a nucleotide source.

\section{Methods}

Sample collection, sequencing, assembly and binning. Groundwater was pumped from an aquifer at the Rifle Integrated Field Research Challenge (IFRC) site, near the town of Rifle, CO, USA at six time points (A-F) between August and 


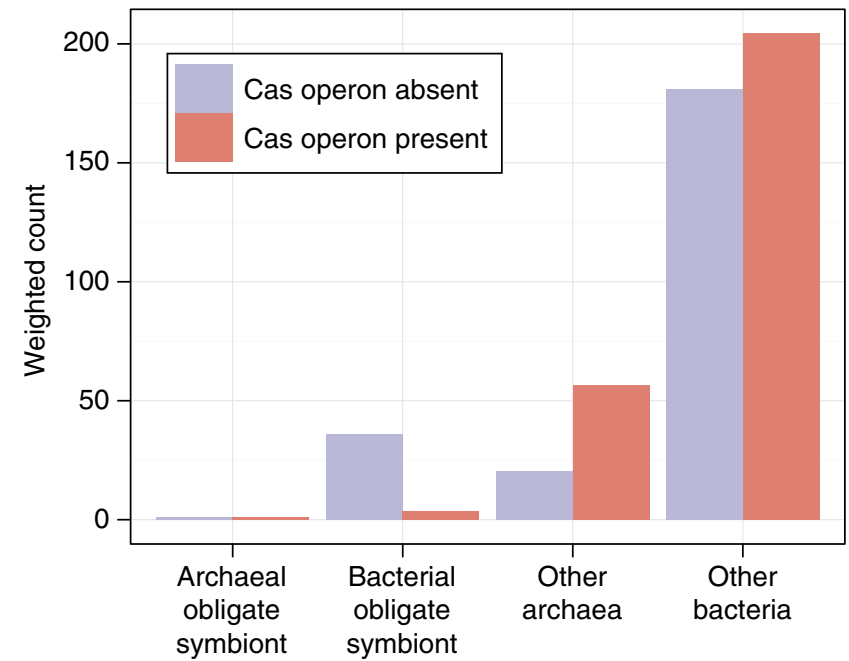

Figure 3 | Association between obligate symbiotic lifestyles and presence of CRISPR-Cas among well-studied bacteria and archaea.

Existence of CRISPR-Cas (purple: absent; red: present) among 1,703 sequenced organisms, including 203 obligate symbionts (two archaea; 201 bacteria) and 1,500 organisms that are not obligate symbionts (164 archaea and 1,336 bacteria). To address sample biases, each genome was assigned a weight inversely proportional to the number of genomes represented in the genus. Thus, each genus received a total count of 1 on the $y$ axis. Even when considering the differences in CRISPR-Cas abundance between bacteria and archaea, a significant association is found between obligate symbiotic lifestyles and presence of CRISPR-Cas ( $P$ value $<1.6 \times 10^{-8}$, ANOVA)

December 2011. Samples were taken before, during and after acetate addition to the aquifer as part of a biostimulation experiment aimed at enriching organisms from little studied $C P^{9-11}$. Groundwater was serially filtered by $1.2,0.2$ and $0.1 \mu \mathrm{m}$ filters. The 0.1 and $0.2 \mu \mathrm{m}$ filters were flash-frozen in a dry ice/ethanol bath on site. DNA was extracted from the filters using the PowerSoil DNA Isolation Kit (MO-BIO), with two modifications to the protocol: DNA elution was done in $50 \mu \mathrm{l}$ Tris buffer; and DNA concentration was done by adding $500 \mu \mathrm{l}$ of $3 \mathrm{M}$ sodium acetate (pH 5.2, adjusted with glacial acetic acid) and $13.75 \mu \mathrm{l}$ of glycogen $\left(20 \mathrm{gl}^{-1}\right)$ to $5 \mathrm{ml}$ of DNA elution, followed by $10 \mathrm{ml}$ of $100 \%$ sterile filtered ethanol.

Extracted DNA was sequenced by the Joint Genome Institute on the Illumina HiSeq 2000 platform producing $150 \mathrm{bp}$ paired-end reads with a targeted insert size of $500 \mathrm{bp}$. All the samples combined produced more than $200 \mathrm{Gbp}$ represented by $\sim 1.6 \times 10^{9}$ reads. Reads were trimmed using Sickle ${ }^{36}$, and then assembled into scaffolds with IDBA_UD ${ }^{37}$. Scaffold coverage was calculated by mapping reads to the assembly using Bowtie2 (ref. 38). Gene prediction was performed by Prodigal $(-\mathrm{p} \text { meta })^{39}$ for all scaffolds longer than $5 \mathrm{kbp}$. Gene annotations were determined based on reciprocal best usearch ( - ublast $)^{40}$ hits against UniRef90 (ref. 41) and $\mathrm{KEGG}^{42}$ databases. One-direction best hit was used when no reciprocal hits were found. Predicted genes were also scanned for known domains and other protein signatures using InterProScan ${ }^{43}$.

Initial clustering of scaffolds into genomic bins was performed using the ABAWACA binning algorithm (https://github.com/CK7/abawaca/tree/master), which used scaffold taxonomic affiliations, time-series abundance patterns, and nucleotide frequencies as measurements to inform binning. Manual refinement of draft genome bins followed. Each bin was evaluated based on coverage, GC content and phylogenetic profile of gene best hits. Genome completeness was evaluated based on inventories of 51 single-copy genes for bacterial genomes ${ }^{12}$, and 54 single-copy genes for archaeal genomes ${ }^{9}$. We recovered a total of 1,724 partial and complete genomes that could be associated to a microbial lineage. The genomes are detailed in Supplementary Data 1.

Crystal Geyser data was assembled as described above from reads of two previously published metagenomic samples acquired from a $\mathrm{CO}_{2}$-driven geyser, located $6 \mathrm{~km}$ south of the town of Green River, Utah, USA, on 8 November 2009 (BioSamples SAMN02419149 and SAMN02419150) ${ }^{44}$. Time-series abundance was determined using additional samples acquired from the site $32 \mathrm{~h}$ earlier (6 November 2009; BioProject PRJNA297582). The scaffolds of the two samples were individually binned using ABAWACA, time-series ESOMs (scaffolds $>3 \mathrm{~kb})^{12}$, and tetranucleotide-frequency based ESOMs (scaffolds $>5 \mathrm{~kb}, 10 \mathrm{~kb}$ window) ${ }^{45}$.

Publically available complete bacterial genomes. Supplementary Data 5 contains the accessions and NCBI Taxonomy IDs for all complete bacterial genomes that were acquired from NCBI's bacterial genomes ftp site (ftp://ftp.ncbi.nih.gov/genomes/Bacteria/).

Viral sequences. Circular viral genomes were identified by searching for scaffolds that could be circularized using paired reads mapped to either end of the scaffold. For each data set we first used Bowtie2 (ref. 38) with parameters '- q -n 2 -e 200 -best' to map the raw reads to all assembled scaffolds longer than $5 \mathrm{kbp}$. Next, we searched for scaffolds whose ends were connected by at least five paired-end reads, and for which no connection to any other scaffold could be identified. Genomes were classified as viral based on existence of viral genes (specified below) and lack of plasmid-specific genes. Genomes that were $\geq 99 \%$ identical over $\geq 99 \%$ of the sequence were considered to belong to the same virus population.

Additional scaffolds were considered viral if they satisfied the following: (a) could not be assigned to any bacterial lineages based on protein similarity; (b) contained two or more viral genes, specifically, ORFs annotated with virus-specific terms ('capsid', 'phage', 'terminase', 'baseplate', 'base plate', 'prohead', 'virion', 'holing', 'virus', 'viral', 'tape measure', 'tapemeasure', 'neck', 'tail', 'p22', 'head', 'T4'); (c) did not include cellular-specific genes (tRNA synthetases, ribosomal protein, preprotein translocases or DNA gyrase subunit A).

Information regarding integrated viral sequence among NCBI completely sequences bacterial genomes was acquired from PHAST ${ }^{46}$ database at http://phast.wishartlab.com/Download.html.

CRISPR-Cas detection. All the microbial draft genomes assembled from Rifle groundwater were scanned for CRISPR arrays using CRISPRfinder ${ }^{47}$, and the resulting arrays were manually curated to filter out false positives. Similarity among retrieved CRISPR repeats was calculated based on all versus all full-length comparison of repeats using 'needle' from the EMBOSS suite ${ }^{48}$. For the heat map presented in Fig. 2, bit scores were normalized based on self-scores. Supplementary Data 3 provides information regarding all the CRISPR arrays identified within the Rifle groundwater genomes.

To identify cas operons, hidden Markov models (HMMs) were constructed for each Cas protein using HMMER 3.1b1 (ref. 49), based on multiple sequence alignments from the NCBI CRISPR/Cas website ${ }^{7}$. These Cas HMMs were used to detect significant hits $\left(\mathrm{E}\right.$-value $<10^{-10}$ ) among the genomes from both Rifle groundwater and NCBI genomes. The presence of Cas1, or alternatively two adjacent ( $\leq 5$ ORFs apart) Cas proteins indicated a cas operon.

To ensure no cas operon was missed due to incorrect gene prediction, six-frame translations of all relevant scaffolds was carried out using 'transeq' from the EMBOSS suite ${ }^{48}$, and all frames were scanned using the Cas1 HMMs. No additional Cas1 proteins were detected by this search, excluding one partial $(<20 \%)$ Cas1 protein, which was found on a contig belonging to an uncharacterized candidate phylum.

We estimated a lower bound for the probability that cas operons were missed due to genome incompleteness based on the average length of cas 1 as follows. First the complete genome length was estimated by dividing the number of recovered nucleotides by the estimated genome completeness (see above). We then calculated the probability for randomly missing a segment of cas 1 length ( 775 nucleotides) given the estimated length of the full genome and the number of nucleotide recovered. This is an underestimation as the length of the CRISPR array was not taken into account and cas operons include several additional proteins.

We tested for association between genome lengths and incidence rates of CRISPR-Cas systems and found that, within each phylum, the presence or absence of CRISPR-Cas could not be explained by the genome length ( $P$ value 0.18 , nested ANOVA, Supplementary Fig. 5).

Phylogenetic analyses. Cas1 phylogeny was based on all Cas1 proteins identified in the Rifle groundwater genomes (scaffolds detailed in Supplementary Fig. 6). Cas9 phylogeny included all the full-length Cas9 proteins available in RefSeq (http://www.ncbi.nlm.nih.gov/refseq/), as well as Cas9 proteins from Rifle metagenomic samples, ORFs and accession numbers are detailed in Supplementary Fig. 7 and Supplementary Data 7. For both Cas1 and Cas9 data sets, amino acid multiple sequence alignments were performed using MAFFT with the 'linsi' scheme $^{50}$, followed by removal of partial proteins. Maximum-likelihood phylogenies were inferred by RAxML ${ }^{51}$ under the PROTGAMMALG evolutionary model with 100 bootstrap re-samplings.

Alternative viral defence mechanism. The number of restriction-modification systems ${ }^{19}$ was determined based on the number of identified restriction enzymes (to avoid over-counting, methylation and specificity subunits were not considered) Restriction enzymes were identified based on either similarity (BLAST e-value $\leq 10^{-10}$, coverage $\geq 80 \%$ ) to restriction enzyme entries of REBASE, the restriction enzyme database ${ }^{52}$, or annotation: considering ORFs including the term 'restriction' in their annotation, but not one of the following terms (as a complete word): 'methyltransferase', 'methylase', 'methylation', 'restriction alleviation', 'antirestriction', 'M', 'mod', 'specificity', 'S', 'McrC', 'hsdS', 'hsdM', 'DptF', 'DptG', 'dam', 'ccrM', 'yhdJ', 'DNMT1', 'E2.1.1.113', 'E2.1.1.72', 'EC:2.1.1.113', 'EC:2.1.1.72', 'ArdA', 'ArdC' and 'lar'. 
Abortive infection systems ${ }^{20}$ were identified based on Abi-coding genes or two toxin-antitoxin coding genes. ORFs with the term 'abortive' or 'abi' were considered Abi-coding genes. Toxin-antitoxin genes were identified based on either BLAST similarity (e-value $\leq 10^{-10}$, coverage $\geq 80 \%$ ) to entries from the toxin-antitoxin database ${ }^{53}$ or on annotation, including ORFs with one of following terms: 'antitoxin', 'anti-toxin', 'antidote-toxin', 'addiction module', 'mazE', 'mazF', 'ChpAK', 'ChpAI', 'mosT', 'mosA', 'yeeV', 'yeeU', 'pasB', 'pasA', 'pemK', 'pis', 'pemI', 'vapB', 'vapC', 'relE', 'relE4', 'stbE', 'prevent-host-death', 'doc', 'phd', 'txe', 'yoeB', 'yefM', 'cogG', 'met]', 'relB', 'dinJ', 'yafQ', 'parE', 'parDB', 'parD1', 'hicA', 'hicB', 'hipA', 'hipB', 'ccdB', 'ccdA'; excluding ORFs whose annotation include: 'topoisomerase', 'cytochrome', 'chemotaxis' or 'motility'.

BREX systems were identified based on the presence of PglZ homologues ${ }^{54,55}$, detected either by their annotation (genes annotated as 'plgZ'), or significant hmmsearch ${ }^{56}$ hit $\left(E-v a l u e ~<10^{-5}\right)$ to Pfam's PglZ HMM (PF08665) ${ }^{57}$

Obligate symbiosis analysis. For the 1,703 organisms with a complete genome in $\mathrm{NCBI}$ and a record in $\mathrm{IMG}^{58}$, we determined whether they are obligate symbionts based on IMG annotation and literature searches (Supplementary Data 7). To detect the significance of the association between the obligate symbiotic lifestyle and the presence of CRISPR-Cas, we used a logistic regression to fit the presence or absence of cas operons to the lifestyle of the microorganism. To account for the large sample bias of available genomes we assigned each genome with a weight inversely proportional to the number of genomes sampled from the same genus.

Nucleotide and fatty acid biosynthesis pathways. Each $\mathrm{KEGG}^{42}$ ortholog group was represented by a HMM: the group's proteins were aligned with MAFFT ${ }^{50}$ and HMMs were constructed using hmmbuild from the HMMER suite ${ }^{56}$. The ORFs from each genome were scanned against the ortholog group HMMs, and were assigned an orthologous group according the best HMM hit (with E-value $<0.01$ ). The completeness of a given biosynthesis pathway was determined based on the number of stages in the pathway for which genes were detected in the genome in question. Nucleotide biosynthesis was considered to be present in a genome if $\geq 75 \%$ of the combined stages of KEGG modules M00048 (Inosine monophosphate biosynthesis) and M00051 (Uridine monophosphate biosynthesis) were satisfied, and it was considered absent if only a third or less of the stages were satisfied (genomes with more than a third but $<75 \%$ of the pathways were considered inconclusive and not taken in to account in this analysis). The fraction of the fatty-acid pathway in a genome was determined based on the number of stages whose genes were detected among KEGG modules M00082 (fatty-acid biosynthesis initiation) and M00083 (fatty-acid elongation).

\section{References}

1. Barrangou, R. et al. CRISPR provides acquired resistance against viruses in prokaryotes. Science 315, 1709-1712 (2007).

2. Garneau, J. E. et al. The CRISPR/Cas bacterial immune system cleaves bacteriophage and plasmid DNA. Nature 468, 67-71 (2010).

3. Sorek, R., Kunin, V. \& Hugenholtz, P. CRISPR-a widespread system that provides acquired resistance against phages in bacteria and archaea. Nat. Rev. Microbiol. 6, 181-186 (2008).

4. Cong, L. et al. Multiplex genome engineering using CRISPR/Cas systems. Science 339, 819-823 (2013).

5. Mali, P. et al. RNA-guided human genome engineering via Cas9. Science 339, 823-826 (2013).

6. Jinek, M. et al. A programmable dual-RNA-guided DNA endonuclease in adaptive bacterial immunity. Science 337, 816-821 (2012).

7. Makarova, K. S. et al. Evolution and classification of the CRISPR-Cas systems. Nat. Rev. Microbiol. 9, 467-477 (2011).

8. Wrighton, K. C. et al. Fermentation, hydrogen, and sulfur metabolism in multiple uncultivated bacterial phyla. Science 337, 1661-1665 (2012).

9. Castelle, C. J. et al. Genomic expansion of domain Archaea highlights roles for organisms from new phyla in anaerobic carbon cycling. Curr. Biol. 25, 690-701 (2015).

10. Luef, B. et al. Diverse uncultivated ultra-small bacterial cells in groundwater. Nat. Commun. 6, 6372 (2015).

11. Brown, C. T. et al. Unusual biology across a group comprising more than $15 \%$ of domain Bacteria. Nature 523, 208-211 (2015).

12. Sharon, I. et al. Time series community genomics analysis reveals rapid shifts in bacterial species, strains, and phage during infant gut colonization. Genome Res. 23, 111-120 (2013)

13. Chylinski, K., Makarova, K. S., Charpentier, E. \& Koonin, E. V. Classification and evolution of type II CRISPR-Cas systems. Nucleic Acids Res. 42, 6091-6105 (2014).

14. Horvath, P. et al. Comparative analysis of CRISPR loci in lactic acid bacteria genomes. Int. J. Food Microbiol. 131, 62-70 (2009).

15. Tyson, G. W. \& Banfield, J. F. Rapidly evolving CRISPRs implicated in acquired resistance of microorganisms to viruses. Environ. Microbiol. 10, 200-207 (2008).
16. Godde, J. S. \& Bickerton, A. The repetitive DNA elements called CRISPRs and their associated genes: evidence of horizontal transfer among prokaryotes. J. Mol. Evol. 62, 718-729 (2006).

17. Jansen, R., van Embden, J. D. A., Gaastra, W. \& Schouls, L. M. Identification of genes that are associated with DNA repeats in prokaryotes. Mol. Microbiol. 43, 1565-1575 (2002).

18. Portillo, M. C. \& Gonzalez, J. M. CRISPR elements in the Thermococcales: evidence for associated horizontal gene transfer in Pyrococcus furiosus. J. Appl. Genet 50, 421-430 (2009).

19. Wilson, G. G. \& Murray, N. E. Restriction and modification systems. Annu. Rev. Genet. 25, 585-627 (1991).

20. Chopin, M. C., Chopin, A. \& Bidnenko, E. Phage abortive infection in lactococci: variations on a theme. Curr. Opin. Microbiol. 8, 473-479 (2005).

21. Krupovic, M., Prangishvili, D., Hendrix, R. W. \& Bamford, D. H. Genomics of bacterial and archaeal viruses: dynamics within the prokaryotic virosphere. Microbiol. Mol. Biol. Rev. 75, 610-635 (2011).

22. Westra, E. R. et al. Parasite exposure drives selective evolution of constitutive versus inducible defense. Curr. Biol. 25, 1043-1049 (2015).

23. Vale, P. F. et al. Costs of CRISPR-Cas-mediated resistance in Streptococcus thermophilus. Proc. R Soc. B 282, 20151270 (2015).

24. Stern, A., Keren, L., Wurtzel, O., Amitai, G. \& Sorek, R. Self-targeting by CRISPR: gene regulation or autoimmunity? Trends Genet. 26, 335-340 (2010).

25. Wyrick, P. B. Intracellular survival by Chlamydia. Cell. Microbiol. 2, 275-282 (2000).

26. Horn, M. et al. Illuminating the evolutionary history of Chlamydiae. Science 304, 728-730 (2004).

27. Kantor, R. S. et al. Small genomes and sparse metabolisms of sedimentassociated bacteria from four candidate phyla. mBio 4, e00708-e00713 (2013)

28. Nelson, W. C. \& Stegen, J. C. The reduced genomes of Parcubacteria (OD1) contain signatures of a symbiotic lifestyle. Front. Microbiol 6, 713 (2015).

29. Pagnier, I. et al. Babela massiliensis, a representative of a widespread bacterial phylum with unusual adaptations to parasitism in amoebae. Biol. Direct 10, 13 (2015).

30. Rinke, C. et al. Insights into the phylogeny and coding potential of microbial dark matter. Nature 499, 431-437 (2013).

31. He, X. et al. Cultivation of a human-associated TM7 phylotype reveals a reduced genome and epibiotic parasitic lifestyle. Proc. Natl Acad. Sci. 112, 244-249 (2015).

32. Iranzo, J., Lobkovsky, A. E., Wolf, Y. I. \& Koonin, E. V. Evolutionary dynamics of the prokaryotic adaptive immunity system CRISPR-Cas in an explicit ecological context. J. Bacteriol. 195, 3834-3844 (2013).

33. Paez-Espino, D. et al. CRISPR immunity drives rapid phage genome evolution in Streptococcus thermophilus. mBio 6, e00262-15 (2015).

34. Levy, A. et al. CRISPR adaptation biases explain preference for acquisition of foreign DNA. Nature 520, 505-510 (2015).

35. Wiens, G. D. et al. Genome sequence of the fish pathogen Renibacterium salmoninarum suggests reductive evolution away from an environmental Arthrobacter ancestor. J. Bacteriol. 190, 6970-6982 (2008).

36. Joshi, N. A. \& Fass, J. N. Sickle: a sliding-window, adaptive, quality-based trimming tool for FastQ files (Version 1.33). Available at https://github.com/ najoshi/sickle (2011).

37. Peng, Y., Leung, H. C. M., Yiu, S. M. \& Chin, F. Y. L. IDBA-UD: a de novo assembler for single-cell and metagenomic sequencing data with highly uneven depth. Bioinformatics 28, 1420-1428 (2012).

38. Langmead, B. \& Salzberg, S. L. Fast gapped-read alignment with Bowtie 2. Nat. Methods 9, 357-359 (2012).

39. Hyatt, D. et al. Prodigal: prokaryotic gene recognition and translation initiation site identification. BMC Bioinformatics 11, 119 (2010).

40. Edgar, R. C. Search and clustering orders of magnitude faster than BLAST. Bioinformatics 26, 2460-2461 (2010).

41. Suzek, B. E., Huang, H., McGarvey, P., Mazumder, R. \& Wu, C. H. UniRef: comprehensive and non-redundant UniProt reference clusters. Bioinformatics. 23, 1282-1288 (2007).

42. Kanehisa, M. \& Goto, S. KEGG: Kyoto encyclopedia of genes and genomes. Nucleic Acids Res. 28, 27-30 (2000).

43. Jones, P. et al. InterProScan 5: genome-scale protein function classification. Bioinformatics 30, 1236-1240 (2014).

44. Emerson, J. B., Thomas, B. C., Alvarez, W. \& Banfield, J. F. Metagenomic analysis of a high carbon dioxide subsurface microbial community populated by chemolithoautotrophs and bacteria and archaea from candidate phyla. Environ. Microbiol.. doi:10.1111/1462-2920.12817 (2015).

45. Dick, G. J. et al. Community-wide analysis of microbial genome sequence signatures. Genome Biol. 10, R85 (2009).

46. Zhou, Y., Liang, Y., Lynch, K. H., Dennis, J. J. \& Wishart, D. S. PHAST: a fast phage search tool. Nucleic Acids Res. 39, W347-W352 (2011).

47. Grissa, I., Vergnaud, G. \& Pourcel, C. CRISPRFinder: a web tool to identify clustered regularly interspaced short palindromic repeats. Nucleic Acids Res. 35, W52-W57 (2007). 
48. Rice, P., Longden, I. \& Bleasby, A. EMBOSS: the European molecular biology open software suite. Trends Genet. 16, 276-277 (2000).

49. Eddy, S. R. Accelerated profile HMM searches. PLoS Comput. Biol. 7, e1002195 (2011).

50. Katoh, K. \& Standley, D. M. MAFFT multiple sequence alignment software version 7: improvements in performance and usability. Mol. Biol. Evol. 30, 772-780 (2013).

51. Stamatakis, A. RAxML version 8: a tool for phylogenetic analysis and post-analysis of large phylogenies. Bioinformatics 30, 1312-1313 (2014).

52. Roberts, R. J., Vincze, T., Posfai, J. \& Macelis, D. REBASE-a database for DNA restriction and modification: enzymes, genes and genomes. Nucleic Acids Res. 43, D298-D299 (2015).

53. Shao, Y. et al. TADB: a web-based resource for Type 2 toxin-antitoxin loci in bacteria and archaea. Nucleic Acids Res. 39, D606-D611 (2011).

54. Goldfarb, T. et al. BREX is a novel phage resistance system widespread in microbial genomes. EMBO J. 34, 169-183 (2015).

55. Makarova, K. S., Wolf, Y. I., Snir, S. \& Koonin, E. V. Defense islands in bacterial and archaeal genomes and prediction of novel defense systems. J. Bacteriol. 193, 6039-6056 (2011).

56. Finn, R. D., Clements, J. \& Eddy, S. R. HMMER web server: interactive sequence similarity searching. Nucleic Acids Res. 39, W29-W37 (2011).

57. Punta, M. et al. The Pfam protein families database. Nucleic Acids Res. 40, D290-D301 (2011).

58. Markowitz, V. M. et al. IMG 4 version of the integrated microbial genomes comparative analysis system. Nucleic Acids Res. 42, D560-D567 (2014).

\section{Acknowledgements}

We thank Andrea Singh for assistance with data management. This research was supported by the US Department of Energy (DOE), Office of Science, Office of Biological and Environmental Research under award number DE-AC02-05CH11231 (Sustainable Systems Scientific Focus Area; Lawrence Berkley National Laboratory operated by the University of California) and award number DE-SC0004918 (Systems Biology Knowledge Base Focus Area). Sequencing was performed at the US DOE Joint
Genome Institute, a DOE Office of Science User Facility, supported under contract DE-AC02-05CH11231. DB was supported by a long-term EMBO fellowship.

\section{Author contributions}

D.B., C.L.S. and J.F.B. designed the study, B.C.T. assembled the data, C.T.B., K.A., A.J.P and J.F.B. reconstructed the genomes, D.B., C.L.S. and I.S. conducted the bioinformatics analyses, D.B. and J.F.B. wrote the manuscript. All authors discussed the results and commented on the manuscript.

\section{Additional information}

Accession codes: Reads and relevant scaffolds from the Rifle Groundwater metagenomes have been deposited in the NCBI BioSample database with accession codes SAMN03202994 to SAMN03203005, and in NCBI BioProject database with accession codes PRJNA298485, PRJNA288027, and PRJNA273161. Crystal Geyser reads and relevant scaffolds are available as part of BioProject PRJNA297582.

Supplementary Information accompanies this paper at http://www.nature.com/ naturecommunications

Competing financial interests: The authors declare no competing financial interests.

Reprints and permission information is available online at http://npg.nature.com/ reprintsandpermissions/

How to cite this article: Burstein, D. et al. Major bacterial lineages are essentially devoid of CRISPR-Cas viral defence systems. Nat. Commun. 7:10613 doi: 10.1038/ncomms10613 (2016).

(c) (i) This work is licensed under a Creative Commons Attribution 4.0 International License. The images or other third party material in this article are included in the article's Creative Commons license, unless indicated otherwise in the credit line; if the material is not included under the Creative Commons license, users will need to obtain permission from the license holder to reproduce the material. To view a copy of this license, visit http://creativecommons.org/licenses/by/4.0/ 